\title{
Synaptophysin Is Required for Synaptobrevin Retrieval during Synaptic Vesicle Endocytosis
}

\author{
Sarah L. Gordon, ${ }^{1}$ Rudolf E. Leube, ${ }^{2}$ and Michael A. Cousin ${ }^{1}$ \\ ${ }^{1}$ Membrane Biology Group, Centre for Integrative Physiology, George Square, University of Edinburgh, EH8 9XD Edinburgh, United Kingdom, and \\ 2Institute of Molecular and Cellular Anatomy, RWTH Aachen University, 52074 Aachen, Germany
}

The integral synaptic vesicle (SV) protein synaptophysin forms $\sim 10 \%$ of total SV protein content, but has no known function in SV physiology. Synaptobrevin (sybII) is another abundant integral SV protein with an essential role in SV exocytosis. Synaptophysin and sybII form a complex in nerve terminals, suggesting this interaction may have a key role in presynaptic function. To determine how synaptophysin controls sybII traffic in nerve terminals, we used a combination of optical imaging techniques in cultures derived from synaptophysin knock-out mice. We show that synaptophysin is specifically required for the retrieval of the pH-sensitive fluorescent reporter sybII-pHluorin from the plasma membrane during endocytosis. The retrieval of other SV protein cargo reporters still occurred; however, their recapture proceeded with slower kinetics. This slowing of SV retrieval kinetics in the absence of synaptophysin did not impact on global SV turnover. These results identify a specific and selective requirement for synaptophysin in the retrieval of sybII during SV endocytosis and suggest that their interaction may act as an adjustable regulator of SV retrieval efficiency.

\section{Introduction}

The localized retrieval and recycling of synaptic vesicles (SVs) after exocytosis is critical for the maintenance of neurotransmission. A key event in this process is the efficient clustering and retrieval of SV proteins from the plasma membrane during endocytosis, which ensures that SVs have the correct molecular composition to participate in the next cycle of neurotransmitter release. The sorting of SV proteins is performed by clathrin adaptor proteins, which recognize specific endocytic cargo motifs (Kelly and Owen, 2011). Not all SV proteins possess such motifs, however, suggesting that other molecules may participate in their retrieval during SV endocytosis.

Synaptobrevin II (sybII) is an integral SV protein that possesses a cytosolic N-terminal tail with an $\alpha$-helical SNARE (soluble NSF attachment protein receptor) motif (Sutton et al., 1998). This motif allows sybII to interact with the plasma membrane SNARE proteins syntaxin and SNAP-25 to drive membrane fusion, resulting in neurotransmitter release (Sudhof, 2004). The cytosolic sybII tail contains noncanonical cargo recognition motifs (Kelly and Owen, 2011), suggesting it may be potentially recognized by classical adaptor proteins or alternately by a distinct adaptor protein. SybII has an established interaction with the integral SV protein synaptophysin (Calakos and Scheller, 1994; Edelmann et al., 1995; Washbourne et al., 1995; Hübner et al.,

\footnotetext{
Received June 22, 2011; revised July 27, 2011; accepted Aug. 15, 2011.

Author contributions: S.L.G. and M.A.C. designed research; S.L.G. performed research; S.L.G. analyzed data; S.L.G., R.E.L., and M.A.C. wrote the paper.

This work is supported by a grant from the Wellcome Trust (088138). We thank Prof. G. Miesenböck, Prof. R. Edwards, and Prof. V. Haucke for the gifts of sybll-pHluorin, vGLUT1-pHluorin, and Syt1-pHluorin, respectively. The authors declare no competing financial interests.

Correspondence should be addressed to Mike Cousin, Membrane Biology Group, Centre for Integrative Physiology, George Square, University of Edinburgh, EH8 9XD Edinburgh, UK. E-mail: M.Cousin@ed.ac.uk.

DOI:10.1523/JNEUROSCI.3162-11.2011

Copyright $\odot 2011$ the authors $\quad 0270-6474 / 11 / 3114032-05 \$ 15.00 / 0$
}

2002). Synaptophysin is an abundant SV protein forming $\sim 10 \%$ of total SV protein content (Takamori et al., 2006); however, studies using synaptophysin knock-out (KO) mice have shown no apparent SV recycling phenotype (Eshkind and Leube, 1995; McMahon et al., 1996). Synaptophysin is proposed to be a chaperone for sybII, controlling either its targeting to SVs (Pennuto et al., 2003; Bonanomi et al., 2007) or its entry into the SNARE complex (Calakos and Scheller, 1994; Edelmann et al., 1995; Becher et al., 1999). It has also been implicated in SV endocytosis, with either dominant-negative approaches (Daly et al., 2000) or gene ablation studies (Spiwoks-Becker et al., 2001) highlighting a potential regulatory role in central nerve terminals.

Since synaptophysin is implicated in both SV endocytosis and sybII targeting to SVs, we hypothesized that synaptophysin could be a potential sybII adaptor protein. To test this hypothesis, we monitored the trafficking and retrieval of the fluorescent reporter superecliptic synaptopHluorin (sybII-pHluorin) in cortical cultures derived from synaptophysin knock-out mice (Eshkind and Leube, 1995). We found that synaptophysin is specifically required for the retrieval of sybII-pHluorin, while its absence slowed the retrieval of other SV protein cargo. Thus synaptophysin is specifically required for sybII retrieval during SV endocytosis.

\section{Materials and Methods}

Materials. SybII-pHluorin, vGLUT1-pHluorin, and synaptotagminpHluorin constructs were provided by Prof. G. Miesenböck (Oxford University, Oxford, UK), Prof. R. Edwards (University of California, San Francisco, CA) and Prof. V. Haucke (Free University of Berlin, Berlin, Germany) respectively. Rabbit anti-sybII antibody was from Abcam. Synaptophysin-mCerulean was generated by replacing EGFP from synaptophysin-EGFP (gift from Jane Sullivan, University of Washington, Seattle, WA) with mCerulean (gift from David Piston, Vanderbilt University, Nashville, TN) using the enzymes AgeI and BsrGI. Neurobasal media, B-27 supplement, penicillin/streptomycin, Minimal Essential 
Medium (MEM), Lipofectamine 2000, AlexaFluor 568 antibody, and FM2-10 were from Invitrogen. All other reagents were from Sigma-Aldrich.

Cortical neuronal cultures. Synaptophysin knock-out mice were maintained as heterozygous breeding pairs, and genotyped as described previously (Schmitt et al., 2009). Dissociated primary cortical neuronal cultures were prepared from E17.5 KO and wild-type embryos of either sex by trituration of isolated cortices to obtain a single-cell suspension, which was plated at a density of 5-10 $\times 10^{6}$ cells/coverslip on poly-Dlysine- and laminin-coated $25 \mathrm{~mm}$ coverslips. Cultures were maintained in Neurobasal media supplemented with B-27, $0.5 \mathrm{~mm}$ L-glutamine, and $1 \% \mathrm{v} / \mathrm{v}$ penicillin/streptomycin. After $72 \mathrm{~h}$, cultures were further supplemented with $1 \mu \mathrm{M}$ cytosine $\beta$-D-arabinofuranoside to inhibit glial proliferation. Cells were transfected after $7 \mathrm{~d}$ in culture with Lipofectamine 2000 according to the manufacturer's instructions, with the following alterations: cells were preincubated in $2 \mathrm{ml}$ of MEM at $5 \% \mathrm{CO}_{2}$ for $30 \mathrm{~min}$ at $37^{\circ} \mathrm{C}$, and then incubated for $2 \mathrm{~h}$ with $2 \mu \mathrm{l}$ of Lipofectamine and $1 \mu \mathrm{g}$ of DNA construct/well. Cells were subsequently washed with MEM before replacement of conditioned Neurobasal media. Cells were imaged after $14-21 \mathrm{~d}$ in culture.

Fluorescent imaging protocols for pHluorin reporters. Cortical cultures were mounted in a Warner imaging chamber with embedded parallel platinum wires (RC-21BRFS) and placed on the stage of a Zeiss Axio Observer D1 epifluorescence microscope. Neurons transfected with synaptophysin-mCerulean were visualized with a $\times 40$ oil-immersion objective at $430 \mathrm{~nm}$ excitation, whereas neurons transfected with pHluorin reporters were visualized at $500 \mathrm{~nm}$ (all $>525 \mathrm{~nm}$ emission). In all experiments, cultures were stimulated with a train of 200 action potentials delivered at $10 \mathrm{~Hz}(100 \mathrm{~mA}, 1 \mathrm{~ms}$ pulse width). Cultures were subjected to continuous perfusion with imaging buffer (in mM: $136 \mathrm{NaCl}, 2.5$ $\mathrm{KCl}, 2 \mathrm{CaCl}_{2}, 1.3 \mathrm{MgCl}_{2}, 10$ glucose, 10 HEPES, pH 7.4). Fluorescent images were captured at $4 \mathrm{~s}$ intervals using a Hamamatsu Orca-ER digital camera and processed offline using ImageJ 1.43 software (NIH). Regions of interest of identical size were placed over nerve terminals that displayed an increase on stimulation and the total fluorescence intensity was monitored over time. All statistical analyses were performed using Microsoft Excel and GraphPad Prism software. The pHluorin fluorescence change was calculated as $\Delta F / F_{0}$ in all cases.

Estimation of surface sybII-pHluorin was performed by perfusing acidic imaging buffer (substituting $20 \mathrm{~mm}$ MES for HEPES, pH 5.5) over cultures for $30 \mathrm{~s}$ (to quench surface sybII-pHluorin) followed by a $1 \mathrm{~min}$ perfusion with standard imaging buffer, $\mathrm{pH}$ 7.4. Cultures were then subjected to alkaline imaging buffer $\left(50 \mathrm{mM} \mathrm{NH}_{4} \mathrm{Cl}\right.$ substituted for $50 \mathrm{~mm}$ $\mathrm{NaCl})$ for $30 \mathrm{~s}$ to reveal total sybII-pHluorin. The surface fraction of sybII-pHluorin as a percentage of total was estimated using the following equation: [(neutral fluorescence - acidic fluorescence)/(alkali fluorescence - acidic fluorescence) $] \times 100$.

Fluorescent imaging protocols for FM2-10 experiments. FM2-10 uptake was evoked with a train of 900 action potentials $(10 \mathrm{~Hz})$. FM2-10 (100 $\mu \mathrm{M})$ was present either during and after stimulation ( $3 \mathrm{~min}$ ) or only after stimulation to label the total SV recycling pool or the poststimulus recycling pool, respectively. Cultures were then washed for $7 \mathrm{~min}$ and dye was unloaded (exocytosis) using a second train of 900 action potentials (10 $\mathrm{Hz}$ ). Neurons transfected with synaptophysin-mCerulean were visualized at $430 \mathrm{~nm}$, whereas FM2-10 unloading was visualized at $500 \mathrm{~nm}$ (both $>525 \mathrm{~nm}$ emission). Responses from nerve terminals were selected from each of the transfected and untransfected neurons in the same field of view. Fluorescence time courses from individual nerve terminals were aligned to the time point before stimulation and averaged. The total fluorescence decrease (SV turnover) was expressed as a percentage of untransfected nerve terminals. For kinetic measurements, the evoked fluorescence decreases were normalized between 1 and 0 and fitted with a single exponential curve.

Immunofluorescence analysis. Synaptophysin knock-out cultures were immunolabeled with anti-sybII antibodies as described previously (Clayton et al., 2009). Neurons transfected with synaptophysin-mCerulean were visualized at $430 \mathrm{~nm}$, whereas sybII immunolabeling was visualized at $550 \mathrm{~nm}$. The diffuseness of sybII was determined by calculating the coefficient of variation (Lyles et al., 2006), where the SD is divided by mean fluorescent intensity across a length of axon.

Statistical analysis. In all cases $n$ refers to the number of independent experiments performed.

\section{Results}

\section{Synaptophysin is required for sybII-pHluorin retrieval from} the plasma membrane

To determine whether synaptophysin was required for sybII retrieval during SV endocytosis, we monitored traffic of the fluorescent reporter sybII-pHluorin in primary cortical cultures derived from either wild-type or synaptophysin knock-out mice. SybII-pHluorin reports the traffic of sybII to and from the cell surface during SV exocytosis and endocytosis, by nature of a $\mathrm{pH}$-sensitive green fluorescent protein (pHluorin) tagged to the luminal domain of sybII (Miesenböck et al., 1998). Its dynamic presence at the cell surface is reported by either increases (exocytosis) or decreases (endocytosis) in its signal, due to its fluorescence being quenched by the acidic environment within SVs.

SybII-pHluorin displayed a punctate distribution when expressed in wild-type neurons, corresponding to its nerve terminal localization (Sankaranarayanan and Ryan, 2000, 2001) (Fig. 1A). However, in synaptophysin knock-out cultures sybII-pHluorin exhibited a more diffuse distribution, suggesting it was mislocalized to the plasma membrane (Fig. $1 B$ ). To confirm the diffuseness of sybII-pHluorin fluorescence, we determined its coefficient of variation $(\mathrm{CV})$ along the axon of transfected neurons. The more diffuse the fluorescence distribution the lower the $\mathrm{CV}$, whereas the more punctate the staining pattern the greater the $\mathrm{CV}$ value (Lyles et al., 2006). As predicted, the CV of sybII-pHluorin fluorescence was significantly smaller in knock-out axons in comparison to either wild-type or knock-out cultures transfected with exogenous synaptophysin-mCerulean (rescued, Fig. $1 C$ ). A parallel decrease in the CV of endogenous sybII also occurred in knock-out neurones (Knock-out CV $47.6 \pm$ 3.4; Rescue $74.8 \pm 3.1$, both $n=4 ; p=0.001$ Student's $t$ test) showing that the absence of synaptophysin affects both exogenous and endogenous sybII (Fig. 1D).

To confirm the mislocalization of sybII-pHluorin to the plasma membrane in knock-out neurons, we determined its surface expression by sequentially applying acidic (to reveal surface expression) and alkaline (to reveal total expression) buffers to wild-type and knock-out cultures (Fig. 1E,F) (Sankaranarayanan and Ryan, 2001). These experiments confirmed that almost $50 \%$ of sybII-pHluorin was present on the cell surface in knockout cultures, in contrast to wild-type [sybII-pHluorin surface expression (\% of total): wild-type $20.8 \pm 2.7 \%, n=3$; knock-out $43.7 \pm 4.0, n=8 ; p=0.009$ Student's $t$ test $]$.

Increased plasma membrane sybII-pHluorin could result from its defective targeting to SVs, increased delivery to the plasma membrane, or inefficient retrieval from the plasma membrane. To delineate between these possibilities, we stimulated either wild-type or knock-out cultures with a train of 200 action potentials $(10 \mathrm{~Hz})$ to trigger SV recycling. A robust stimulationdependent increase in sybII-pHluorin fluorescence occurred in synaptophysin knock-out cultures, indicating that the reporter is efficiently delivered to the cell surface by SV exocytosis (Fig. 2A). However sybII-pHluorin fluorescence remained elevated once stimulation was terminated in knock-out neurons, in contrast to wild-type neurons, which decayed back to baseline with firstorder kinetics (Fig. 2A). The rate of sybII-pHluorin fluorescence decay after stimulation contains contributions from both SV endocytosis and acidification (Sankaranarayanan and Ryan, 2000). 
A
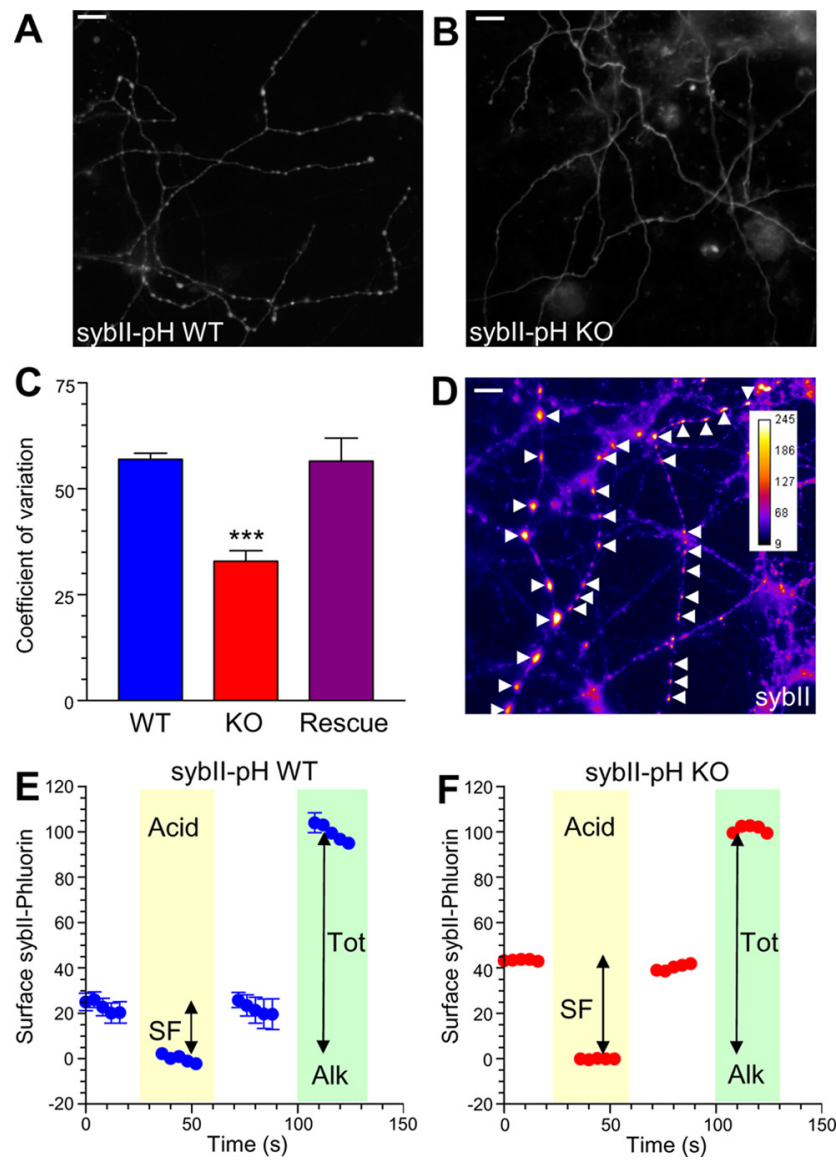

Figure 1. Sybll-pHluorin is stranded on the cell surface in synaptophysin knock-out neurons. Representative images show the differential expression of sybll-pHluorin in either wild-type (WT, $\boldsymbol{A})$ or synaptophysin KO (B) cultures. Scale bar represents $15 \mu \mathrm{m}$. $\boldsymbol{C}$, Bar graph displays the coefficient of variation of sybll-pHluorin fluorescence along axons of WT, KO, and rescued neurons in alkaline buffer. Data are presented as $\pm \mathrm{SEM}, n=6$ for WT, $n=5$ for KO, $n=4$ for rescue, ${ }^{* * *} p<0.001$ one-way ANOVA for K0 against both WT and rescue. $\boldsymbol{D}$, Representative image of the distribution of endogenous sybll using immunofluorescence in $\mathrm{KO}$ cultures transfected with synaptophysin-mCerulean. Sybll distribution in the rescued neuron is highlighted by arrowheads (all other neurons in field of view are K0). False coloring indicates the intensity of sybll immunolabeling (calibration bar displayed). Scale bar represents $10 \mu \mathrm{m}$. $\boldsymbol{E}, \boldsymbol{F}$, Representative traces displaying the proportion of sybll-pHluorin expressed on the cell surface in either WT (blue circles, $\boldsymbol{E}$ ) or KO (red circles, $\boldsymbol{F}$ ) neurons. Sybll-pHluorin fluorescence is normalized to $100 \%$ in alkaline buffer (green bars) and to $0 \%$ in acidic buffer (yellow bars). Arrows indicate either surface fraction (neutral pH - acidic pH, SF) or total sybll-pHluorin (alkaline pH - acidic $\mathrm{pH}$, Tot).

Since SV acidification is unaltered in synaptophysin knock-out cultures (Kwon and Chapman, 2011), this indicates that the sustained elevation in sybII-pHluorin fluorescence after stimulation is due to its defective retrieval from the plasma membrane.

To confirm that the defect in sybII-pHluorin retrieval was due to the absence of synaptophysin, we expressed exogenous synaptophysin-mCerulean in knock-out cultures. This resulted in a complete rescue of sybII-pHluorin retrieval kinetics (Fig. $2 B$ ). Thus synaptophysin is required for the retrieval of sybIIpHluorin from the nerve terminal plasma membrane.

Deletion of synaptophysin slows retrieval of other SV protein cargo, but global SV turnover is unaltered

We next determined whether synaptophysin was required for the retrieval of other SV protein cargo, or whether the requirement is specific to sybII. To achieve this, we examined the retrieval of two independent pHluorin-tagged reporters of SV protein cargo, the
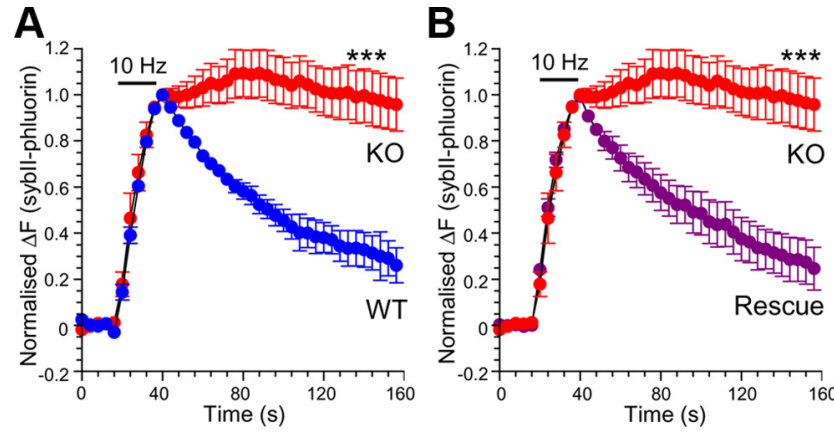

Figure 2. Synaptophysin is required for sybll-pHluorin retrieval. Sybll-pHluorin transfected wild-type (WT, blue circles), synaptophysin KO (red circles), or KO neurons expressing synaptophysin-m(erulean (Rescue, purple circles) were stimulated with a train of 200 action potentials $(10 \mathrm{~Hz}$, indicated by bar). Averaged traces for either WT and $\mathrm{KO}(\boldsymbol{A})$ or $\mathrm{KO}$ and Rescue (B) are displayed \pm SEM, $n=10$ for WT, $n=8$ for K0, $n=9$ for rescue, ${ }^{* * *} p<0.001$ two-way ANOVA for KO against both WT and rescue.
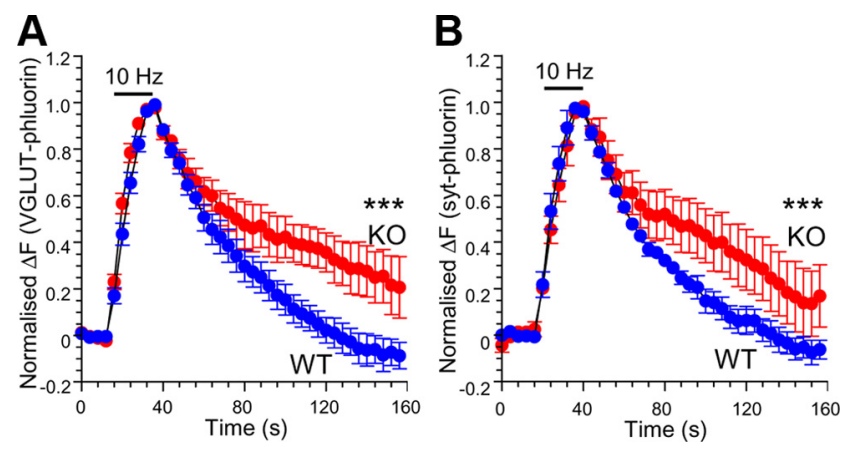

Figure 3. Synaptophysin is not required for retrieval of other SV protein cargo. Wild-type (WT, blue circles) and synaptophysin KO (red circles) cultures were transfected with either vGLUT-pHluorin $(\boldsymbol{A})$ or syt-pHluorin $(\boldsymbol{B})$. Cultures were stimulated with a train of 200 action potentials $(10 \mathrm{~Hz}$, indicated by bar). Averaged traces are \pm SEM,$n=8$ for WT vGLUT-pHluorin, $n=4$ for all other conditions, ${ }^{* * *} p<0.001$ two-way ANOVA for WT against KO for both vGLUT-pHluorin and syt-pHluorin.

vesicular glutamate transporter (vGLUT-pHluorin) and synaptotagmin (syt-pHluorin) (Diril et al., 2006; Voglmaier et al., 2006), in wild-type and knock-out neurons. When expressed in wild-type neurons, both reporters were retrieved with first-order kinetics after a train of 200 action potentials (Fig. $3 A, B$ ). vGLUTpHluorin and syt-pHluorin were also retrieved in synaptophysin knock-out cultures, albeit with slower kinetics when compared against wild-type (Fig. $3 A, B$ ). Thus the general retrieval of SV protein cargo is slowed in the absence of synaptophysin.

We next determined whether the slowing of SV protein cargo retrieval in synaptophysin knock-out cultures translated into a global defect in SV turnover. To achieve this, we examined the loading and unloading of FM2-10, a fluorescent dye that labels SVs during endocytosis (Clayton et al., 2009). A second stimulus of FM2-10-loaded neurons reports both the extent of SV turnover (total unload) and rate of SV exocytosis (unloading kinetics). There was no significant difference in the extent of SV turnover (endocytosis followed by exocytosis) between knockout and rescued neurons for either the total SV recycling pool (\% of knock-out: knock-out $100 \pm 7.2$, rescue $103.7 \pm 9.6, n=3$, $p=0.64$ Student's $t$ test; Fig. $4 C$ ) or the pool of SVs that were retrieved after stimulation (\% of knock-out: knock-out $100 \pm$ 4.9 , rescue 102.5 $\pm 3.5, n=4, p=0.69$ Student's $t$ test; Fig. $4 D$ ). Knock-out and rescued neurons also had identical FM2-10 unloading kinetics, indicating no role for synaptophysin in SV exo- 


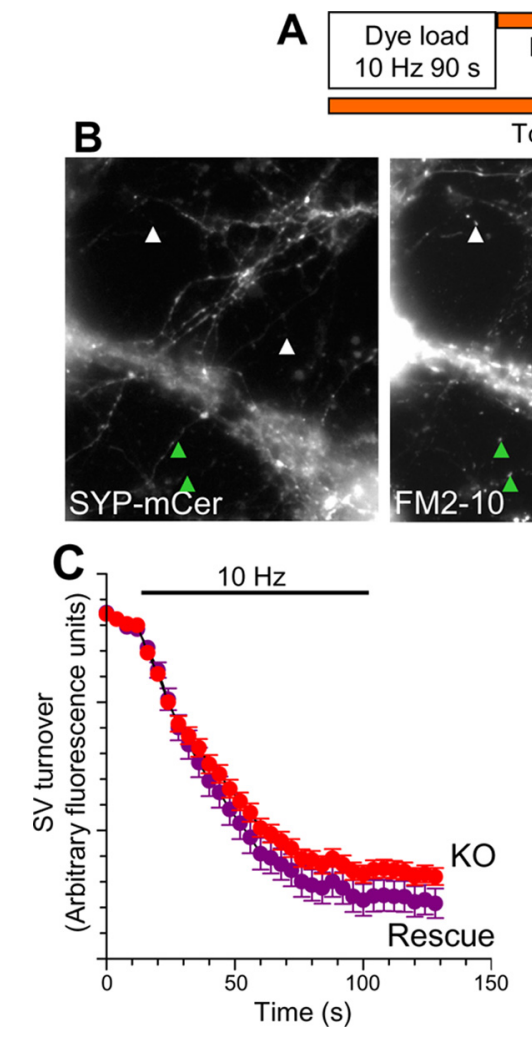

Post-stimulus pool (D)

Total recycling pool (C)

B


Figure 4. Global SV turnover is normal in synaptophysin knock-out neurons. $A$, Synaptophysin $\mathrm{KO}$ cultures were transfected with synaptophysin-mCerulean (Rescue) and loaded with FM2-10 (100 $\mu \mathrm{m})$ using a train of 900 action potentials $(10 \mathrm{~Hz})$. FM2-10 was applied either during and after stimulation to load the total $S V$ recycling pool or only after stimulation (to load the poststimulus recycling SV pool). B, Representative images display synaptophysin-m(erulean transfected neuron (left panel), FM2-10 loading (middle panel) and merged image (right panel). Loading in $\mathrm{KO}$ neurons indicated by white arrowheads and in rescued neurons by green arrowheads. Scale bar represents $20 \mu \mathrm{m} . \mathbf{C}, \boldsymbol{D}$, Representative traces display dye unloading in $\mathrm{KO}$ (red circles) and rescued (purple circles) neurons evoked by 900 action potentials $(10 \mathrm{~Hz}$, indicated by bar) in the same field of view for either the total recycling SV pool (C) or the poststimulus SV recycling pool (D).

cytosis ( $\tau$-knock-out $28.8 \pm 3.1 \mathrm{~s}$, rescue $31.1 \pm 3.4, n=3$ independent experiments, $p=0.77$ Student's $t$ test). Thus while SV protein cargo retrieval is slowed in synaptophysin knock-out neurons, the number of SVs that are turned over is unchanged.

\section{Discussion}

We have shown that synaptophysin is specifically required for the retrieval of sybII, but not other SV protein cargo from the nerve terminal plasma membrane during endocytosis. This is the first direct functional evidence of a molecular role for synaptophysin in SV physiology.

We observed a mislocalization of both sybII-pHluorin and endogenous sybII to the plasma membrane in synaptophysin knock-out neurons. In agreement, overexpression of sybII in either neurons or a heterologous expression system resulted in a similar phenotype, a defect that was corrected by transfection of exogenous synaptophysin (Pennuto et al., 2003; Bonanomi et al., 2007). Increased surface sybII could result from defective targeting during SV biogenesis, its increased delivery to the plasma membrane, or its disrupted retrieval from the plasma membrane. Our data suggest that the first two possibilities can be discounted, since first, sybII-pHluorin still reports SV exocytosis (indicating that it is correctly targeted to SVs), and second, SV exocytosis itself is unaltered in knock-out mice (McMahon et al., 1996; Janz et al., 1999; Kwon and Chapman, 2011; our observations). Thus, the mislocalization of sybII-pHluorin in synaptophysin knock-out neurons results from a defect in its recovery during SV endocytosis, as evidenced by the lack of retrieval after its delivery to the plasma membrane by exocytosis.

The synaptophysin-sybII interaction occurs in resting nerve terminals and is decreased during neuronal activity (Prekeris and Terrian, 1997; Pennuto et al., 2002; Reisinger et al., 2004; but see Khvotchev and Südhof, 2004), suggesting that stimulation frees sybII from synaptophysin to participate in SV exocytosis (Calakos and Scheller, 1994; Edelmann et al., 1995; Becher et al., 1999). Further examination showed that calcium influx is a key event in disrupting this complex (Prekeris and Terrian, 1997; Daly and Ziff, 2002; Reisinger et al., 2004), suggesting that manipulation of intracellular free calcium levels may also influence sybII retrieval. SybII interacts with synaptophysin via its transmembrane region (Yelamanchili et al., 2005), indicating the local lipid microenvironment may also be critical in determining the strength of their interaction. In agreement, depletion or enhancement of the cholesterol membrane content resulted in decreased or increased complex formation respectively (Mitter et al., 2003). Some factors do not influence the stability of the sybII-synaptophysin complex, however. For example, protein kinase or phosphatase antagonists do not alter complex levels in vivo, suggesting that the phosphorylation of the cytoplasmic tails of either protein does not influence their interaction (Khvotchev and Südhof, 2004). Indeed the presence of the cytosolic synaptophysin $\mathrm{C}$ terminus is not required for its binding to syb II (Bonanomi et al., 2007; Felkl and Leube, 2008). Therefore it seems likely that the interaction between sybII and synaptophysin occurs via their transmembrane domains, with formation of the complex determined by both the local lipid microenvironment and neuronal activity. Future experiments will focus on these parameters to determine how sybII retrieval can be manipulated during SV endocytosis.

We found that the absence of synaptophysin had no global effect on SV turnover, in agreement with previous studies (Eshkind and Leube, 1995; McMahon et al., 1996; Janz et al., 1999). Recent work has shown that SV endocytosis is slowed in synaptophysin knock-out neurons (Kwon and Chapman, 2011), which is consistent with our observation of a decrease in retrieval kinetics of both vGLUT-pHluorin and syt-pHluorin. The impact of this slowing in terms of SV endocytosis is small, however, with a reduction in endocytic capacity of only $\sim 10 \%$ for a defined time period (Kwon and Chapman, 2011). This explains why we did not observe a significant difference in the extent of global SV turnover between synaptophysin knock-out and rescued neurons. Thus, while the absence of synaptophysin has minor effects on SV endocytosis kinetics, the major consequence of its deletion is the arrest of sybII retrieval from the plasma membrane. Interestingly, a similar slowing of SV endocytosis is reported in sybII knock-out neurons (Deák et al., 2004), strongly suggesting that 
the endocytic phenotype observed in synaptophysin knock-out neurons is due to defective sybII retrieval.

Other isoforms of syb such as sybIII and sybIV possess acidic dileucine cargo recognition motifs that allow their recognition by the endosomal adaptor proteins AP-3 and AP-1, respectively (Darsow et al., 1998; Peden et al., 2001). This motif is not conserved in sybII, suggesting that it may not be recognized by the plasma membrane adaptor protein AP-2. In contrast, the synaptophysin $\mathrm{C}$ terminus contains a large number of potential tyrosine-based cargo motifs, which are classic AP-2 interaction sites. Therefore synaptophysin may act as a molecular bridge between classical adaptor proteins and sybII to allow efficient retrieval of the latter (Felkl and Leube, 2008). Experiments are currently in progress to test this hypothesis and to determine how both the structure and molecular properties of synaptophysin control sybII retrieval.

Note added in proof. Genetic deletion of synaptogyrin in $C$. elegans also results in mislocalization of sybII from nerve terminals (Abraham et al., 2011).

\section{References}

Abraham C, Bai L, Leube RE (2011) Synaptogyrin-dependent modulation of synaptic neurotransmission in Caenorhabditis elegans. Neurosci 190: 75-88.

Becher A, Drenckhahn A, Pahner I, Margittai M, Jahn R, Ahnert-Hilger G (1999) The synaptophysin-synaptobrevin complex: a hallmark of synaptic vesicle maturation. J Neurosci 19:1922-1931.

Bonanomi D, Rusconi L, Colombo CA, Benfenati F, Valtorta F (2007) Synaptophysin I selectively specifies the exocytic pathway of synaptobrevin 2/VAMP2. Biochem J 404:525-534.

Calakos N, Scheller RH (1994) Vesicle-associated membrane protein and synaptophysin are associated on the synaptic vesicle. J Biol Chem 269:24534-24537.

Clayton EL, Anggono V, Smillie KJ, Chau N, Robinson PJ, Cousin MA (2009) The phospho-dependent dynamin-syndapin interaction triggers activity-dependent bulk endocytosis of synaptic vesicles. J Neurosci 29:7706-7717.

Daly C, Ziff EB (2002) Ca2+-dependent formation of a dynaminsynaptophysin complex: potential role in synaptic vesicle endocytosis. J Biol Chem 277:9010-9015.

Daly C, Sugimori M, Moreira JE, Ziff EB, Llinás R (2000) Synaptophysin regulates clathrin-independent endocytosis of synaptic vesicles. Proc Natl Acad Sci U S A 97:6120-6125.

Darsow T, Burd CG, Emr SD (1998) Acidic di-leucine motif essential for AP-3-dependent sorting and restriction of the functional specificity of the Vam3p vacuolar t-SNARE. J Cell Biol 142:913-922.

Deák F, Schoch S, Liu X, Südhof TC, Kavalali ET (2004) Synaptobrevin is essential for fast synaptic-vesicle endocytosis. Nat Cell Biol 6:1102-1108.

Diril MK, Wienisch M, Jung N, Klingauf J, Haucke V (2006) Stonin 2 is an AP-2-dependent endocytic sorting adaptor for synaptotagmin internalization and recycling. Dev Cell 10:233-244.

Edelmann L, Hanson PI, Chapman ER, Jahn R (1995) Synaptobrevin binding to synaptophysin: a potential mechanism for controlling the exocytotic fusion machine. EMBO J 14:224-231.

Eshkind LG, Leube RE (1995) Mice lacking synaptophysin reproduce and form typical synaptic vesicles. Cell Tissue Res 282:423-433.

Felkl M, Leube RE (2008) Interaction assays in yeast and cultured cells confirm known and identify novel partners of the synaptic vesicle protein synaptophysin. Neuroscience 156:344-352.

Hübner K, Windoffer R, Hutter H, Leube RE (2002) Tetraspan vesicle membrane proteins: synthesis, subcellular localization, and functional properties. Int Rev Cytol 214:103-159.

Janz R, Südhof TC, Hammer RE, Unni V, Siegelbaum SA, Bolshakov VY (1999) Essential roles in synaptic plasticity for synaptogyrin I and synaptophysin I. Neuron 24:687-700.
Kelly BT, Owen DJ (2011) Endocytic sorting of transmembrane protein cargo. Curr Opin Cell Biol 23:404-412.

Khvotchev MV, Südhof TC (2004) Stimulus-dependent dynamic homoand heteromultimerization of synaptobrevin/VAMP and synaptophysin. Biochemistry 43:15037-15043.

Kwon SE, Chapman ER (2011) Synaptophysin regulates the kinetics of synaptic vesicle endocytosis in central neurons. Neuron 70:847-854.

Lyles V, Zhao Y, Martin KC (2006) Synapse formation and mRNA localization in cultured Aplysia neurons. Neuron 49:349-356.

McMahon HT, Bolshakov VY, Janz R, Hammer RE, Siegelbaum SA, Südhof TC (1996) Synaptophysin, a major synaptic vesicle protein, is not essential for neurotransmitter release. Proc Natl Acad Sci U S A 93:4760-4764.

Miesenböck G, De Angelis DA, Rothman JE (1998) Visualizing secretion and synaptic transmission with $\mathrm{pH}$-sensitive green fluorescent proteins. Nature 394:192-195.

Mitter D, Reisinger C, Hinz B, Hollmann S, Yelamanchili SV, Treiber-Held S, Ohm TG, Herrmann A, Ahnert-Hilger G (2003) The synaptophysin/ synaptobrevin interaction critically depends on the cholesterol content. J Neurochem 84:35-42.

Peden AA, Park GY, Scheller RH (2001) The di-leucine motif of vesicleassociated membrane protein 4 is required for its localization and AP-1 binding. J Biol Chem 276:49183-49187.

Pennuto M, Dunlap D, Contestabile A, Benfenati F, Valtorta F (2002) Fluorescence resonance energy transfer detection of synaptophysin I and vesicle-associated membrane protein 2 interactions during exocytosis from single live synapses. Mol Biol Cell 13:2706-2717.

Pennuto M, Bonanomi D, Benfenati F, Valtorta F (2003) Synaptophysin I controls the targeting of VAMP2/synaptobrevin II to synaptic vesicles. Mol Biol Cell 14:4909-4919.

Prekeris R, Terrian DM (1997) Brain myosin V is a synaptic vesicleassociated motor protein: evidence for a $\mathrm{Ca}^{2+}$-dependent interaction with the synaptobrevin-synaptophysin complex. J Cell Biol 137:1589_ 1601.

Reisinger C, Yelamanchili SV, Hinz B, Mitter D, Becher A, Bigalke H, AhnertHilger G (2004) The synaptophysin/synaptobrevin complex dissociates independently of neuroexocytosis. J Neurochem 90:1-8.

Sankaranarayanan S, Ryan TA (2000) Real-time measurements of vesicleSNARE recycling in synapses of the central nervous system. Nat Cell Biol 2:197-204.

Sankaranarayanan S, Ryan TA (2001) Calcium accelerates endocytosis of vSNAREs at hippocampal synapses. Nat Neurosci 4:129-136.

Schmitt U, Tanimoto N, Seeliger M, Schaeffel F, Leube RE (2009) Detection of behavioral alterations and learning deficits in mice lacking synaptophysin. Neuroscience 162:234-243.

Spiwoks-Becker I, Vollrath L, Seeliger MW, Jaissle G, Eshkind LG, Leube RE (2001) Synaptic vesicle alterations in rod photoreceptors of synaptophysin-deficient mice. Neuroscience 107:127-142.

Sudhof TC (2004) The synaptic vesicle cycle. Annu Rev Neurosci 27:509-547.

Sutton RB, Fasshauer D, Jahn R, Brunger AT (1998) Crystal structure of a SNARE complex involved in synaptic exocytosis at $2.4 \AA$ resolution. Nature 395:347-353.

Takamori S, Holt M, Stenius K, Lemke EA, Grønborg M, Riedel D, Urlaub H, Schenck S, Brügger B, Ringler P, Müller SA, Rammner B, Gräter F, Hub JS, De Groot BL, Mieskes G, Moriyama Y, Klingauf J, Grubmüller H, Heuser J, et al. (2006) Molecular anatomy of a trafficking organelle. Cell 127:831-846.

Voglmaier SM, Kam K, Yang H, Fortin DL, Hua Z, Nicoll RA, Edwards RH (2006) Distinct endocytic pathways control the rate and extent of synaptic vesicle protein recycling. Neuron 51:71-84.

Washbourne P, Schiavo G, Montecucco C (1995) Vesicle-associated membrane protein-2 (synaptobrevin-2) forms a complex with synaptophysin. Biochem J 305:721-724.

Yelamanchili SV, Reisinger C, Becher A, Sikorra S, Bigalke H, Binz T, AhnertHilger G (2005) The C-terminal transmembrane region of synaptobrevin binds synaptophysin from adult synaptic vesicles. Eur J Cell Biol 84:467-475. 